XXII.

\title{
Ueber Eröfinung des Wirbelcanales bei Spondylitis und Compressionsmyelitis.
}

\author{
Von \\ Prof. Fürstner \\ in Strassburg i. E. \\ (Hierzu Tafel XIV.)
}

\begin{abstract}
$W_{\text {ährend die Zahl der Gehirnerkrankungen, bei denen Erfolg ver- }}$ sprechende chirurgische Eingriffe in Betracht gezogen werden können, den anfangs zu hoch gespannten Erwartungen gegenüber eine beträchtliche Einschränkung erfahren hat, war das Gebiet der Rückenmarkskrankheiten, bei denen an chirurgische Hilfe zu denken war, von vornherein ein verhältnissmässig kleines, und ausserdem war die Frequenz der in diese Gruppe gehörenden Fälle weitaus geringer als die der therapentisch gleichwerthigen cerebralen Affectionen. In erster Linie war ja daran zu denken, bei den spinalen Erkrankungen operativ vorzugehen, die durch Druckwirkung bedingt waren, sei es, dass letztere durch Knochentheile, durch Nenbildungen oder durch Blut und anderweitige Flüssigkeitsansammlungen ausgeübt wurde. Da nun die Drucklähmungen in Folge von Fracturen oder Geschwulstbildung doch immerhin selten sind, so musste sich das Hauptinteresse der Beobachter vor allem der Beantwortung der Frage zuwenden, ist auch bei den weitaus häufigeren und practisch wichtigeren, spinalen Erkrankungen, die im Gefolge einer Spondylitis auftreten, ein operativer Eingriff gerechtfertigt und Erfolg: versprechend. Diese Erwägung war um so begreiflicher angesichts der Erfahrung, dass bei vielen derartigen einschlägigen Fällen trotz aller aufgewendeter Mühe, trotz aller für den Behandelnden und den Kranken gleich beschwerlicher Lagerungsversuche ein Fortschreiten des Processes nicht aufzuhalten ist, dass andere Fälle über-
\end{abstract}


haupt erst in späten Stadien, wenn besonders schon Störungen der Blasenfunction vorhanden und Lagerungsversuche kaum noch Erfolg versprechen, zur Behandlung kommen, dass endlich aber angesichts der zunehmenden Schwere der Krankheitserscheinungen ein nahezu passives Verhalten für den behandelnden Arzt immer weniger erträglich wird. Dazu kommt, dasss man sich von der Ausbreitung und Zugänglichkeit des Krankheitsheerdes leicht ein zu günstiges Bild entwirft. Kraske*) hat die verschiedenartigen anatomischen Veränderungen, die hier in Betracht kommen können, klargestellt und unter Berücksichtigung derselben die Zulässigkeit und die Chancen eines operativen Eingriffes erörtert. Seine Erfahrungen über den Erfolg des letateren sind weitaus weniger ermuthigend wie die von Macewen berichteten, jedenfalls bält er bei der grossen Majorität der Fälle, besonders bei allen denen, wo der Wirbelkörper Sitz des Krankheitsheerdes, operatives Vorgehen nur dann zulässig, wenn die sonstigen Behandlangsmethoden erfolglos, wenn andererseits die Erscheinungen sich steigern und vor Allem auch Blase und Mastdarm mitbetheiligt sind. Immerhin ist das Material, über das Kraske verfügte, verhältnissmässig gering; er weist auch selbst auf den Umstand hin, dass wir umfangreichere, nach allen Richtıngen hin. zuverlässige statistische Mittheilungen über den Verlauf derartiger Fälle bei exspectativer Behandlung nicht besitzen; das Ergebniss einer grösseren Zahl von spontanen Besserungen oder gar Heilungen würde gewiss zusammen mit den anderweitigen Bedenken den Entschluss operativ vorzugehen, noch mehr erschweren.

Aus den Darlegungen Kraske's, denen ich durchaus beistimmen muss, geht schon hervor, dass der anatomische Status in den einzelnen Fällen ein sehr verschiedenartiger sein, dass die diagnostische Beurtheilung desselben auf grosse Schwierigkeiten stossen kann. Wenn Urban*) das operative Vorgehen bei Spondylitis tuberculosa nur für zulässig erachtet, wenn der Process seit Monaten zum Stillstand gekommen, wenn keine Abscesse vorhanden, so wird über diese Vorbedingung in manchen Fällen überhaupt nicht sicher zu urtheilen sein. Noch weniger gelingt es meist, sich ein klares Bild zu machen von der Art und Weise, wie die Druckerscheinungen zu Stande kommen und noch weniger von der Qualität und Ausbreitung der Veränderungen im Rückenmark. Endlich besitzen wir keine Anhaltspunkte für die Entscheidung: der Frage, sind die im Rückenmark gesetzten Degenerationen und die dadurch bedingten Functionsstörungen überhaupt noch einer Rück-

*) Archiv für klinische Chirurgie Bd. 41 .

**) Verhandlungen der deutschen Gesellschaft für Chirurgie. 1892. 
bildung fähig, wie weit sind schon die secundären Degenerationen vorgeschritten.

Der nachstehend mitgetheilte Fall scheint mir einmal bezüglich des operativen Vorgehens bei durch Spondylitis bedingten Drucklähmungen einen nicht unwichtigen Beitrag zu liefern, weiter hat er Werth für die Beurtheilung der Beziehungen, die zwischen Traumen und Spondylitis bestehen, und endlich bieten die bei der mikroskopischen Untersuchung des Rückenmarks gefundenen Veränderungen manches Interessante, soweit es sich um die Ausbreitung der secundären Degenerationsvorgänge, um die Druckwirkung selbst und um die Veränderungen handelt, die erst nach der Operation entstanden sind.

Paulin, Maria, 35 Jahre alt, war zur Tuberculose - soweit festzustellen - nicht hereditär disponirt, beide Eltern leben, sind gesund, sie selbst will immer wohl und kräftig gewesen sein, niemals bestand besonders Husten und Auswurf. Aus einer Ehe mit einem geisteskrank gestorbenen Manne stammt ein gesundes Kind. Ein Jahr vor der Aufnahme schweres Trauma, Patientin stürzte vom Heuboden herunter, 3 Meter tief auf den Rücken, und zwar auf einen Haufen Steine' aufschlagend. Anfangs bestanden an der getroffenen Rückenpartie heftige Schmerzen, die aber wieder verschwanden, so dass Patientin ihre Arbeit in der Fabrik aufnehmen konnte. Fast 6 Monate nach dem Trauma begannen ron Neuem die Schmerzen, nach dem Nacken und der rechten Bauchseite zu ausstrahlend, gleichzeitig stellte sich Schwäche und etwas Steifigkeit in beiden Beinen ein, ebenso war das Gefühl an den unteren Extremitäten herabgesetzt. Nach 2 Monaten war die Schwäche so beträchtlich, dass Patientin nicht mehr gehen lionnte, die Empfindung war völlig aufgehoben. Im untersten Theil der Brustwirbelsäule bei Druck Schmerzen, allmälig soll hier der Knochen hervorgetreten sein. Der Stuhlgang war dauernd angehalten, erfolgte nur noch auf Medicamente. Der Urin musste sehr häufig gelassen werden, seit 5-6 Wochen Incontinenz, Patientin merkte auch den Wasserabgang schliesslich nicht mehr. Allmälige Abmagerung.

Aufnahme in die Klinik am 26. Juli 1894.

Status. Gracile, schlecht genährte Fran, viel älter aussehend als sie ist. An Kopf und den oberen Extremitäten keinerlei Abnormität, im Bereich der Lungen nirgends Dämpfung, in den Lungenspitzen, besonders links, ist das Exspirium etwas verschärft und verlängert, ganz vereinzelte Rasselgeräusche hörbar. Sputum nicht zu erhalten. An der Herzspitze systolisches Geräusch, zweiter Pulmonalton verstärkt. Die Wirbelsäule zeigt in ihren oberen Theilen keinerlei Difformität, im Bereich des 8. bis 10. Brustwirbels springen die Processus spinos. gibbusartig hervor, bei intensivem Druck und bei Klopfen wird hier Schmerz geäussert. Totale Paraplegie beider Beine, keine degenerative Atrophie der Muskeln, letztere leicht rigide." Patellarreflexe hochgradigst gesteigert, Dorsalclonus, Plantarreflexe sehr lebhaft, Bauchreflex nur in den unteren Partien auszulösen. Totạle Empfindungslähmung für alle Qualitäten bị 
zu einer Linie, die zwischen den beiden Spin. anter. inferior. gelegt wird, und zwar besteht die Empfindungslähmung gleichmässig um den ganzen Körper. Oberhalb dieser Linie kommt eine etwa bis zum Nabel reichende Zone, in der die Empfindung, namentlich hinten, gleichfalls in geringerem Grade herabgesetzt ist.

Vollständige Incontinentia resicae, fast unauf hörliches l'räufeln.

Urin trübe alkalisch, deutlicher Befund für eine bestehende Cystitis.

Abdominalorgane ohne Veränderung. Hartnäckige Obstipation. Nirgends Anschwellung der Drüsen.

Im Bereich der linken Hälfte des Gesässes beginnender Decubitus. Abends Temperatursteigerung.

Schon in den nächsten Tagen dehnte sich trotz aller Mittel der Decubitus weiter aus, auch auf der rechten Hinterbacke derbe Infiltration und Verfärbnug der Haut. Ebenso gelang es nicht, den Blasencatarrh zu bessern; die Reaction des Urins war deutlich alkaliseh, Eiter, Blutkörper, Epithelien reichlich nachweisbar. Die Abendtemperatur war regelmässig erhöht bis $39^{\circ}$ und mehr, anchMorgens an einzelnen Tagen Fieber.

Die diagnostische Beurtheilung dés Falles war zunächst insofern schwierig, als nicht die ätiologischen Beziehungen klar lagen, die etwa zwischen dem Trauma und den Krankheitserscheinungen bestanden. Da für eine anderweitige Erkrankung der Wirbelsäule, da namentlich für eine tuberculöse Spondylitis irgendwie sichere Anhaltspunkte nicht vorlagen - die ganz geringen Erscheinungen seitens der Lunge konnten als solche wohl nicht angesehen werden -, da das Trauma ein zweifellos schweres gewesen, da unmittelbar nach demselben intensive Schmerzen an der getroffenen Stelle bestanden hatten, die nach mehrmonatlicher Besserung sich dann von Neuem einstellten, und in ihrem Gefolge Symptome der Parese, da an der getroffenen Stelle der Wirbelsäule immerhin eine Difformität bestand, wenn auch von geringen Dimensionen, so schien mir die Annahme gerechtfertigt, dass das Trauma die eigentliche Ursache der spinalen Erkrankung gewesen. Welche Veränderungen aber durch dasselbe an den Wirbeln, den Hänten oder gar dem Mark gesetzt worden seien, darüber erschien mir ein sicheres Urtheil unmöglich, das Auftreten schwerer spinaler Symptome erst 6 Monate nach dem Trauma glaubte ich aber in dem Sinne deuten zu können, dass das Rückenmark jedenfalls erst secundär in Mitleidenschaft gezogen worden sei. Damit war aber die Möglichkeit gegeben, dass nach Entfernung eines etwaigen irritirenden Knochenfragmentes oder einer epiduralen Veränderung, das Rückenmark wieder functionsfähig werden könnte. Bei dem ungünstigen Ernährangszustand der Kranken, bei Berücksichtigung der vergeblichen Versuche, den Blasenkatarrh zu bessern, das Fortschreiten des Decubitus aufzuhalten, bei dem consumirenden Fieber 
erschien die Prognose nicht zweifelhaft, es musste in Kurzem ein letaler Ausgang befürchtet werden; dabei war die Patientin oft von den heftigsten Schmerzen bei Tag und Nacht gequält. Unter diesen Umständen erschien mir ein Versuch, durch operatives Eingreifen Klarheit zu schaffen und eventuell Besserung der Erscheinungen za erreichen, gerechtfertigt, und wir entschlossen uns im Einverständniss mit der Kranken und ihren Angehörigen zur Operation.

Dieselbe wurde in der hiesigen chirurgischen Klinik von Professor F. Fischer ausgeführt. Da letzterer über den Verlauf derselben, soweit es sich um die chirurgischen Momente bandelt, an anderer Stelle zu berichten beabsichtigt, beschränke ich mich auf das Nothwendigste.

In linker Seitenlage wurde auf der Höhe des Gibbus ein etwa $15 \mathrm{Ctm}$. langer Schnitt gefïhrt, die Proc. spinosi des 8., 9., 10. Brustwirbels werden freigelegt und abgemeisselt, ebenso die Bögen und so das Rückenmark in einer Austehnung von $3-4 \mathrm{Ctm}$. freigelegt. Die Dura ist stark gespannt, sieht blass aus. Eine Compression des Marks, eine Einengung des Wirbelcanals etwa durch Granulationsgewebe ist nirgends mit Sicherheit festzustellen, das Mark pulsirt nicht. Bei Lagerung der Kranken, den Angaben Chipault's gemäss, wird die Dura gespalten, die vollkommen unverändert glatt, glänzend erscheint, das blass aussehende Rückenmark drängt sich etwas vor, aber auch jetzt ist bei vorsichtig ausgeführtem Umfühlen des Markes nirgends eine Compression wahrnehmbar, auch am Knochen, besonders auch an der vorderen Wand des Wirbelcanals ist nichts Abnormes zu entecken; die Wunde wurde mit Jodoformgaze ausgestopft und mit Knopfnaht rerschlossen. Der Heilungsprocess ging ohne Störung von Statten, 20 Tage nach der Operation war die Narbe fest verschlossen.

Der operative Eingriff hatte also weder Veränderungen an der Wirbelsäule, noch am Rückenmark und seinen Häuten klargelegt mit Ausnahme der fehlenden Pulsation des Markes.

Der weitere Verlauf bot manches Bemerkenswerthe. Zunächst besserte sich die Function der Blase, während der Urin bis dahin ständig abtröpfelte, vermochte die Kranke am dritten Tage nach der Operation den Urin ziemlich vollständig zu halten, so dass sie mehrere Male catheterisirt werden und während der Zwischenzeit den Uxin halten Ironnte, sodann war die Steigerung der Patellarreflexe wesentlich geringer besonders rechts und endlich besserte sich das Empfindungsvermögen in dem Grade, dass drei Tage nach der Operation allerdings ziemlich starke Reize rechts von 3, links von 2 Finger unter dem Poupart'schen Bande an deutich wahrgenommen wurden.

Am zehnten Tage wurde die Sensibilitätsgrenze durch die Mitte des Oberschenkels markirt, Patientin verspürte anch deutlich Abgang von Urin und Stuhl, am 17. Tage wurden Reize schon von etwas oberhalb der Malleolen an wahrgenommen und meist auch richtig localisirt. Später trat am Rumpf und besonders deutlich an den Armen eher Hyperästhesie hervor. An den Beinen war 
auch die Temperaturempfindung wieder gekehrt; die Schmerzempfindung war jedenfalls nicht erheblich gemindert. Die früheren Schmerzen waren vollkommen verschwunden.

Absolut unverändert blieb aber die Lähmung der Beine. Auch die Cystitis bestand freilich in geringerem Grade fort, trotzdem die Blase zwei Mal täglich mit 3 proc. Borsäurelösung ausgespült wurde, der Decubitus breitete sich, nach vorübergehender Besserung, beiderseits aus. Die Intensität der Patellarreflexe und zwar beiderseits, nahm immer mehr ab, bei passiven Bewegungen machte sich im Bereich der Unterextremitäten noch leichte Spannung bemerkbar. Obwohl das subjective Befinden ein gutes war, keinerlei Klagen über Schmerzen oder Sensationen bestanden, die Nahrungsaufnahme eine reichliche war - übrigens bei sehr hartnäckiger Obstipation - ging der Ennährungszustand der Patientin immer mehr zurück.

Abends fast regelmässig Fieber. Aussehen des Decubitus bald besser, bald schlechter. Im Bereich der linken Lungenspitze verschärftes Exspirium noch deutlicher, vereinzelte Rasselgeräusche, nirgends Dämpfung oder bronchiales Athmen. Mehrere Tage bestanden heftige Diarrhoen, dann wieder Verstopfung:

Bei ständigem hohem Fieber, Fortbestehen von Cystitis, von Decubitus, hochgradiger Verfall. Sensorium gelegentlich benommen, die Angaben der Kranken sehr schwankend. An den unteren Extremitäten, die auf's äusserste abgemagert, nur insofern eine Aenderung, als die Patellarreflexe nicht mehr auszulösen sind. Am 13. October etwa 6 Wochen nach der Operation plötzlicher Collaps, am 14. October Exitus.

Die Obduction (Prof. v. Recklinghausen) ergab einen in mehrfacher: Hinsicht iberraschenden Befund, den ich nur, soweit es sich um Wirbelsäule und Rückenmark handelt, in extenso wiedergebe:

Am unteren Theile der Brustwirbelsäule lineare, 9 Ctm. lange weissliche Narbe, die Haut mit der Unterlage fest verwachsen, im mittleren Theile ebenso Dura mit dem Knochen. Im unteren Theile des etwas weiten Duralsackes klare gelbe Flüssigkeit. Im oberen Theile Dura mit Arachnoidea mehrfach leicht verklebt und letztere mehr flächenhaft getrübt. Im unteren Brustmark entsprechend der Hautnarbe haftet das Rückenmark den Wirbelkörpern fest an, nach der Lösung findet sich an der hinteren Fläche der Wirbelkörper entsprechend dem 9. Brustwirbel eine linöcherne Prominenz, die in den Wirbelcanal 4 bis $5 \mathrm{Mm}$. hineinragt, unmittelbar nach dieser stufenförmigen Prominenz ist der nun folgende Theil der Wirbelsäule mehr nach vorn gelagert. Auf der knöchernen Prominenz lagern weiche käsige Massen von geringer Diclie, ein flaches Polster, von demselben weichen Material gebildet, befindet sich in einer Ausdehnung von $3 \mathrm{Ctm}$. auf der entsprechenden Durapartie. In dieser Deckschicht findet sich ein kleiner Knochensequester. Entsprechend der käsigen Auflagerung Verdickung der Dura, auf der Innenfläche lebhafte Injection und mehrfache strangförmige Verklebungen derselben mit der weichen Haut, letztere selbst an dieser Stelle netzförmig getrübt und verdickt. Das Rückenmark zeigt an der Stelle der Stufe eine Einschnürung und Verjüngung, der Breitendurch- 
messer hier und an den benachbarten Partien differirt fast um $2 \mathrm{Mm}$. Die Consistenz des Rückenmarkes ist in diesem Theile scheinbar nicht verändert: Bei einem Schnitte oberhalb der Einschnürungsstelle keine deutlichen Verfärbungen erkennbar, nach dem Halsmark zu, und in diesem heben sich die Goll'schen Stränge zum Theil durch grauere Verfärbung ab, eine gleiche findet sich nahezu in dreieckiger Form unter der Compressionsstelle in den Seitensträngen. Die Basis ist nach der Peripherie gerichtet.

Wie meist am frischen Rückenmark sind diese Farbendifferenzen nicht constant, sie ändern. sich etwas je nach der Beleuchtung.

lch lasse nun gleich den Befund folgen, der sich nach Entfernung der Brust- und Bauchorgane ergiebt.

Von der Höhe des 6. Brustwirbels an erscheint die Wirbelsäule von vorn gesehen nach beiden Seiten hin stark aufgetrieben, die Anschwellung fluctuirt. Beim Finreissen entleert sich reichliche dickeitrige Flüssigkeit. Nach unten reicht die Auftreibung rechts bis zum unteren Rand des ersten Lendenwirbelkörpers, links nur bis zum-12. Brustwirbels. Die Vorderfläche des ersten Lendenwirbels ist frei, vor der des 12. Brustwirbels eine Eiter enthaltende Auftreibung, die rechts in den seitlichen Sack übergeht. Im Grunde dieses Theiles des Abscesses liegt der Körper des 12. Brustwirbels frei zu Tage. Der Abscess reicht bis zum 6. Brustwirbel. An Stelle des 10 . Brustwirbels findet sich eine grosse Eiterhöhle, die nach unten von einer rauhen Knochenfläche begrenzt ist, oben durch die schon erwähnte, in den Wirbelcanal prominirende Leiste, den Rest des 9. Brustwirbel. Nach Aufsägen der Wirbelkörper in sagittaler Richtung zeigt sich ein weiterer käsiger Herd im Körper des 7. und 12. Wirbels, die Bandscheiben zum grössten Theil zerstört.

Es ist also der 10. Brustwirbel total, der 9. partiell eingeschmolzen, der Rest des letzteren prominirt in den Rückenmarkscanal, der Körper des elften Brustwirbels ist nach vorn verschoben, weitere Herde bergen die Körper der 7. und 12. Brustwirbel.

Im Hirn ergab sich eine beginnende basilare tuberculöse Meningitis, zwei symmetrisch gelegene kleine Cysten in den Streifenhügeln.

Im Herzen an der Mitral- und Tricuspidalklappe zahlreiche kleine warzige Verdichungen.

Im oberen Lappen der linken Lunge eine kirschgrosse subpleural gelegene Caverne mit dickem käsigen Inhalt, kleine bronchopneumonische Herde im Gewebe zerstreut. In der rechten Lunge finden sich diese durchweg kleinen Herde in noch grösserer Zahl. Keine miliaren Knötchen.

Links: Pyelonephritis. Hochgradige Cystitis.

Bevor ich mich den mikroskopischen Veränderungen zuwende, die sich im Rückenmarke fanden, möchte ich auf das Missverhältniss hinweisen, das zwischen dem Befunde bei der Operation und den Ergebnissen der Obduction besteht. Da der Einschnitt entsprechend dem 8 . bis 10. Brustwirbel erfolgte, da wir nach Freilegung des Rückenmarkes in diesem Abschnitte keinerlei Anomalien entdecken konnten, 
auch bei der Betastung nach vorn zu nicht, vor Allem keine Einengungen des Wirbelcanals, keine Knickungen des Marks, bleiben nur die Möglichkeiten, dass die Veränderungen in der Ausdehnung, die die Obduction ergab, zur Zeit des operativen Eingriffes überhaupt noch wicht bestanden, dass sie sich also verhältnissmässig schnell entwickelt haben, oder dass sie sich der Feststellung bei Lebzeiten auf irgend eine Weise entzogen. Die Krankheitssymptome sprachen dafür, dass vor der Operation ein Druck auf das Rückenmark stattfand, es war wenigstens Monate hindurch das klinische Bild der Compressionsmyelitis nachweisbar; im weiteren Verlanf bildete sich ein Theil der Störungen, vor Allem die sensiblen zurïck, neue, die im Sinne einer Vermehrung des Druckes gesprochen hätten, traten überhaupt nicht auf; diese Momente dürften doch in dem Sinne aufzufassen sein, dass zur Zeit des operativen Eingriffes und vor demselben der Druck eher stärker war als nach demselben. Znnächst habe ich mich nun bei der Operation des Eindruckes nicht erwehren können, dass die genaue Betastung und Untersuchung der vorderen Fläche des Wirbelcanals, also der Rückwand der Wirbelkörper bei in sito befindlichem Rückenmark ziemlich schwierig ist, besonders schwer zu palpiren werden die Wirbelkörper sein, wenn die Freilegung des Markes nur in geringen Dimensionen erfolgte. Aus diesem Grunde könnte es auch bei Operationen am Rückenmark, analog wie bei manchen chirurgischen Eingriffen am Schädel zweckmässig sein, den Schnitt und die Freilegung des Markes in möglichst grossen Dimensionen vorzunehmen, auf der anderen Seite werden aber durch ein solches Vorgehen die Gefahren einer nachträglichen Difformität der Wirbelsäule noch gesteigert, ein Uebelstand, der vielleicht gemindert würde durch die von Urban vorgeschlagene Operationsmethode, bei der möglichst viel Knochen erhalten wird. Jedenfalls können wohl zur Zeit der Operation noch nicht bestanden haben die Prominenz des Restes des 9. Brustwirbels in den Canal hinein, und ebenso dürfte wohl die Einschmelzung des 10. Brustwirbels noch nicht soweit vorgeschritten und ein Eindringen des an seine Stelle getretenen Abscesssackes in den Wirbelkanal noch nicht stattgefunden haben. Immerhin fehlte bei der Freilegung des Markes die Pulsation und hatte sich auch bei Beendigung der Operation nicht hergestellt. Andererseits fehlten aber auch Veränderungen an den Rückenmarkshäuten, insbesondere war die Dura intact. Wenn nun aber die Einschnürung des Markes durch den prominirenden Rest des 9. Wirbelkörpers zur Zeit der Operation noch nicht bestehen konnte, so wird die Frage auf Grund des makroskopischen Befundes schwer zu beantworten sein, wodurch wurden die Symptome der Druckmyelitis bedingt? Wohl bestand im Beginn ein Symptom, das 
nach Kraske besonders häufig bei epiduralen Exsudaten getroffen wird, intensive, ausstrahlende Schmerzen, die auf eine Mitbetheiligung der Wurzeln hinweisen, wo soll aber zur Zeit derOperation ein Exsudat localisirt gewesen sein? Wenn nun aber ein Exsudat bei der Operation nicht nachweisbar war, andererseits eine Compression bestand, so wird man daran denken müssen, dass im Körper des neunten und zehnten Brustwirbels wohl schon wesentliche Veränderungen vorhanden waren, an denen auch die Bandscheiben participirten. Diese Veränderungen können sehr wohl Circulationsstörungen und Blutungen in den Lymphwegen bedingt haben, die dann ihrerseits zu Veränderungen in der Marksubstanz führten und den Effect der Compression hervorriefen. Dass diese Anomalien auch nach der Eröffnung des Wirbelcanals weder für die Inspection noch Palpation erkenubar waren, dass man scheinbar auf normale Verhältnisse stiess, scheint mir ganz besonders wichtig zu sein. Vielleicht kann in analogen Fällen, das Fehlen der Pulsation des Markes von diagnostischem Werth sein.

Die hier entstehenden Schwierigkeiten sprechen gewiss zu Gunsten der von Kraske vertretenen Anschauung, dass es oft kaum möglich sein wird, bei der Operation sich ein zutreffendes Bild von der Ausdehnung des destructiven Processes zu machen, dass es andererseits erst recht unmöglich ist, dem Krankheitsheerde, wenn er im Wirbelkörper zu suchen, erfolgreich therapentisch beizukommen. Der Umstand, dass im vorliegenden Falle immerhin eine vorübergehende Besserung erreicht, dass meiner Ueberzeugung nach das Leben um einige Wochen verlängert wurde, dürfte nicht genügend sein, um die Chancen eines operativen Eingriffes in analogen Fällen günstiger erscheinen zu lassen. Wird doch andererseits nicht mit Sicherheit auszuschliessen sein, dass durch die Operation, durch die Veränderung der Druckverhältnisse ein schnelleres Fortschreiten des Processes, besonders in den Wirbelkörpern, bedingt worden ist. Schwierig ist nun weiter die Frage zu entscheiden, welche Beziehungen zwischen dem Trauma und der tuberkulösen Erkrankung bestehen. Die Kranke und ihre Angehörigen haben mit Bestimmtheit versichert, dass vor dem Falle keinerlei Störungen bestanden, es ist jedenfalls kein einziges Moment nambaft dafür zu machen, dass etwa vor dem Fall schon eine Erkrankung der Wirbelsäule bestand, es wird deshalb nichts übrig bleiben, als anzunehmen, dass durch das Trauma directe Verletzungen des Knochens nicht gesetzt wurden, dass aber der in den Wirbelknochen schon latent bestehende Process durch das Trauma gesteigert wurde in einem Grade, dass nach 6 Monaten die ersten Symptome bemerkbar wurden.

Die bei der Operation gesetzte Wunde heilte ohne jeden $Z$ wischen 
fall, auch dies wäre wohl kaum möglich gewesen, wenn schon zur Zeit der Operation die Knochenveränderungen ausgedehnte gewesen wären; die Verwachsungen, die sich zwischen der Dura und weichen Haut gebildet, waren nur unbedeutender Art. Im Krankheitsverlauf nach der Operation war zunächst bemerkenswerth die schnelle Besserung der Sensibilität, auch bei den von Kraske operirten Fällen wurde dieselbe günstige Aenderung beobachtet, die in einzelnen Fällen sogar nach Verlauf von Stunden schon eintrat. Die letzten Lebenstage ausgenommen, war bei unserer Kranken nur eine geringe Sensibilitätsstörung an den unteren Extremitäten nachweisbar, die Reize mussten etwas kräftiger sein, und die Localisation war nicht absolut zuverlässig, dafür stellte sich am Rumpf und besonders in den Oberextremitäten eher ein gewisser Grad von Hyperästhesie ein. Vollkommen unverändert blieb die Lähmung. Eine Besserung der Blasenfunction war insofern zu constatiren, als die beständige Incontinenz beseitigt wurde, die Cystitis bestand aber fort, und ebenso konnte ein Weiterschreiten des Decubitus nicht gehindert werden. Sehr wesentlich modificirt wurde endlich das Verhalten der Patellarreflexe. Sehr bald nach der Operation nahm die bis dahin hochgradige Steigerung derselben ab und zwar zuerst rechts, dann links, bei passiven Bewegungen des Unterschenkels war gleichzeitig noch leichte Spannung zu überwinden, allmälig verschwanden die Patellarreflexe beiderseits, während die Hautreflexe, namentlich der Plantarreflex bis zum Tode der Kranken lebhaft blieben.

Das Rückenmark wurde in der gewohnten Weise gehärtet, und unter Anwendung der Weigert'schen, der Doppelfärbung Eosin-Hämatoxylin und der v. Gieson'schen Methode gefärbt. Leider ging von der Höhe, die der Prominenz, also dem 9. Brustwirbel entsprach, ein ganz kleiner Theil des Markes, das hier doch in seiner Consistenz mehr verändert war, als es bei der Obduction schien verloren; vom 8. Brustwirbel an war die Härtung gelungen.

Zunächst ergab die Untersuchung das Vorhandensein einer Meningitis, die durchweg frischer Art abwärts von der Operationsstelle mehr an der hinteren Fläche des Markes, aufwärts mehr auf der vorderen entwickelt war. Neben hochgradiger Infiltration der Haut selbst mit Rundzellen, neben der gleichen Veränderung, die sich in den Wandungen der grösseren Gefässe nachweisen liess, waren an einer Reihe von Stellen auch die in das. Mark eintretenden Gefässe von einem mehr oder weniger breiten Saum von Rundzellen umgeben, und ebenso liess sich an circumscripten Stellen eineRan dinfiltration des Markes erkennen. Hier waren auch die Axencylinderquellungen besonders deutlich. Veränderungen älterer Art waren an den Häuten nirgends nachzuweisen, das- 
selbe gilt auch, wie ich gleich erörtern werde, zum Theil für das Mark selbst.

An dem Theile, der nach oben zu am nächsten der Compressionsstelle lag, liess sich unregelmässig vertheilt durch die ganze Breite des Markes Degeneration erkennen. Die Zeichnung der grauen Substanz war undeutlich, zum Theil ganz fehlend.

Was nun die anf- und absteigende Degeneration angeht, so musste auf die Gestaltung derselben der Umstand immerhin einen gewissen Einfluss ausüben, dass die Compressionsstelle verhältnissmässig tief lag in der Höhe des 9. Wirbelkörpers; vor Allem war zu erwarten, dass die aufsteigende Degeneration sich auf kleinere Abschnitte erstrecken würde, als es bei dem weitans häufigeren Sitz der Compression in der Mitte oder gar im oberen Theile der Brustwirbelsäule der Fall zu sein pflegt.

In der Höhe des 8. Wirbelkörpers findet sich noch eine ausgedehnte Erkrankung fast der gesammten Seiten- und Hinter- in geringerem Grade auch der Vorderstränge bei ganz unregelmässiger Vertheilung, dann treten nach oben zu allmälig die typischen Degenerationsfiguren hervor. Die graue Substanz hebt sich stellenweis noch undentlich ab. Was zunächst die Hinterstränge angeht, so fällt auf, dass durchweg am stärksten verändert sind die äusseren Partien der Degenerationsfigur, and zwar in den Höhen, wo die Burdach'schen Stränge noch mitbetroffen sind, ebenso aber im Halsmark ausschliesslich im Bereich der Goll'schen Stränge. Innerhalb der letzteren, von denen ein ziemlich breiter peripher gelegener Saum überhaupt nicht degenerirt, lässt sich dann nach innen zu ein besonders stark verändertes Gebiet und endlich der hinteren Längsspalte in Gestalt eines Dreiecks mit der Spitze nach oben anliegend, ein weiterer Abschnitt constatiren, wo die Degeneration geringfügiger, wo jedenfalls noch zahlreiche intacte Nervenfasern liegen. Die Betheiligung der Burdach'schen Stränge ist nicht bedentend, auch hier fällt die stärkere Erkrankung in den peripheren Theilen auf. Im Halstheil bleibt dann die Spitze des erkrankten Dreiecks, das einem Theil der Goll'schen Stränge entspricht, allmälig immer mehr von der hinteren Commissur zurück. Denkt man sich die gesammten Goll'schen Stränge in drei Theile zerlegt, so sind im Halsmark etwa Zweidrittel verändert; denkt man sich die von der hinteren Commissur bis zur Peripherie reichende Entfernung in drei Theile zerlegt, so liegt die Spitze der Degenerationsfigur von der hinteren Commissur gerechnet am Ende des ersten Drittels.

Was die Stärke der secundären degenerativen Processe angeht, so lässt sich nicht verkennen, dass dieselbe weitaus beträchtlicher ist da, wo es sich um absteigende Degeneration handelt, dies gilt für die Hin- 
terstränge ebenso wie für die Kleinhirnseitenstrangbahnen und das Gower'sche Bündel.

Was nun die ausser den Goll'schen Strängen aufsteigend degenerirten Bahnen anbetrifft, so sind sofort nach Aufhören der mehr diffusen Veränderungen zwei von einander trennbare Züge an der Peripherie der Seitenstränge zu unterscheiden. Einmal eine veränderte Zone, die mit breiter Basis die graue Substanz des Hinterhorns berührt, die sich allmälig verjüngend bis etwa an die Mitte der Seitenstränge reicht; hier beginnt dann zunächst schmal eine weitere Degenerationsfigur an der Peripherie des Seitenstranges, in der Höhe der vorderen Grenze des Vorderhornes sich zu einer dreieckigen Figur verbreiternd, deren Basis nach der Peripherie zu gelegen ist; allmälig nimmt die Breite dieses degenerirten Saumes wieder ab, derselbe ist aber bis weit in das Ge. biet der vorderen Warzeln, d. h. bis weit in die Vorderstränge hinein zu verfolgen. Es dürfte wohl keinem Zweifel unterliegen, dass die erste Degenerationsfigur den Kleinhirnseitensträngen, dass die zweite dem Gowers'schen Bündel entspricht.

An mehreren Präparaten, namentlich an der Grenze des oberen Brustmarkes, habe ich den Eindruck gewonnen, dass diese beiden Degenerationsfiguren thatsächlich von einander getrennt sind durch einen allerdings ganz kleinen Bezirk normalen Gewebes, ich halte es aber nicht für ansgeschlossen, dass bei den nach der Weigert'schen Methode gefertigten Präparaten ganz geringe Veränderungen nicht mit Deutlichkeit hervortreten, ich muss also die Möglichkeit zulassen, dass an die Degenerationsfigur der Kleinhirnseitenstränge, und zwar an die vordere Spitze derselben unmittelbar die des Gowers'schen Bündels stiess. Was den Grad der Degeneration in beiden Bündeln anbetrifft, so war derselbe in den Kleinhirnseitensträngen eher geringer als im Gowers'schen Bündel, auch hierbei dürfte der verhältnissmässig tiefe Sitz der Compressionsstelle und die Integrität eines grösseren Theiles der Clarke'schen Säulen in Betracht kommen. Nach oben zu wurde die Degenerationsfigur der Kleinhirnseitenstrangbahn immer kleiner, während im obersten Theil des Brustmarkes und im untersten Theil des Cervicalmarkes die dreieckige Figur, die dem Gowers'schen Bündel angehört, immer breiter hervortritt.

Im obersten Halsmark ist die letztere Degeneration noch zu verfolgen, die Kleinhirnseitenstrangdegeneration hebt sich nicht mehr so deutlich ab. Bei der Unsicherheit, die noch über die Gestaltung und Grösse des Gowers'schen Bündels herrscht, ist jedenfalls bemerkenswerth, dass die Degeneration desselben einmal soweit nach unten zu verfolgen, dass sie ferner im obersten Theil des Halsmarkes noch ziemlich 
kräftig war und dass sie sich unmittelbar an die vordere Grenze der Degenerationsfigur der Kleinhirnseitenstrangbahn anlegt. Die weitere Verfolgung des Bündels durch die Medulla oblongata wurde durch einen anglücklichen Zufall gehindert. Klinische Symptome, die im Sinne einer mehr oder weniger starken Betheiligung der einen oder anderen Bahn zu verwerthen wären, sind in unserem Falle nicht nambaft zu machen.

Dagegen lässt der verschiedene Grad der Degeneration, wie er in den Goll'schen Strängen geschildert wurde, vielleicht die Gestaltung der sensiblen Störungen, wie sie vor und nach der Operation sich darboten, verständlich erscheinen, es steht der Annahme nichts im Wege, dass ganz abgesehen von den überhaupt intact gebliebenen Fasern andere ihre Functionsfähigkeit wieder erlangt haben, die ihnen vorübergehend in Folge der Druckverhältnisse genommen war.

Eine analoge Ausgleichfähigkeit bezüglich der sensiblen Störungen ist, wie ich oben bemerkte, auch in anderen Fällen beobachtet worden.

Wie in einzelnen Fällen Kraske's, so blieb dagegen auch in dem vorliegenden die Motilitätsstörung vollkommen unbeeinflusst, in Uebereinstimmung damit war die absteigende Degeneration in den Pyramidenseitenstrangbahnen weit intensiver als die aufsteigende; es scheint also in derartigen Fällen durch einen operativen Eingriff viel eher eine günstige Aenderung der sensiblen als der motorischen Störungen erreichbar zu sein. Sehr bemerkenswerth ist bei unserer Kranken gewiss das Schwinden der Schmerzen trotz der frischen Meningitis, trotz - allerdings geringer - Veränderung einzelner hinterer Wurzeln!

Was nun die Degeneration unterhalb der Compressionsstelle angeht, so finden sich auch hier eine Reihe Abweichungen von den typischen Befunden. Ich habe schon hervorgehoben, dass im Bereich der Pyramidenbahnen die Veränderungen besonders intensiv waren, hier fanden sich auch allein vorgerücktere Stadien des degenerativen Processes, auffallend ist sodann, dass sich nach vorn mit breiter Basis an die Pyramidenseitenstränge anstossend, beiderseits ein peripher gelegener Degenerationsstreifen constatiren lässt, der sich nach vorn zu immer mehr verschmälert und im Niveau der vorderen Grenze des Vorderhorns aufhört. Dabei ist in diesem Abschnitt die Degeneration entschieden weniger stark, als in den Pyramidensträngen selbst. Es würde hier also ausser der typischen absteigenden Pyramidenseitenstrangdegeneration, noch ein weiterer Abschnitt der Seitenstränge der Degeneration anheimgefallen sein. Um welche Bahnen es sich hier handelt, vermag ich nicht zu entscheiden.

Endlich findet sich aber auch in den Hintersträngen eine Degeneration, die nicht sehr intensiv, aber bis weit in das Lumbalmark 
hinein zu verfolgen ist, die mit Ausnahme des bekannten Saumes, der dem Hinterhorn parallel läuft, und des peripheren Abschnittes fast die ganze Breite der Hinterstränge einnimmt, rechts etwas mehr als links, und die allmälig sich immer mehr verschmälert. Der degenerative Process in den Hintersträngen ist offenbar ein wenig alter; wie er zu denten, muss zunächst wohl dahingestellt bleiben; mit der bekannten commaförmigen Degenerationsfigur in den Hintersträngen verglichen, ist er auffallend in die Breite gehend. In dem klinischen Bilde dürfte er meiner Ansicht nach zum Ausdruck gekommen sein in der Aenderung, welche sich bezüglich der Patellarreflexe constatiren liess. Bald vach der Operation minderte sich die Steigerung derselben, and zwar erheblicher auf der rechten Seite, schliesslich waren sie überhaupt nicht mehr auszulösen.

Ueberblicken wir noch einmal die spinalen Degenerationen, so waren auch sie jedenfalls ausgedehnter, als es zu erwarten war, ferner ist es schwierig, den Zeitpunkt zu bestimmen, in dem sie zur Entwickelung gelangt sind. Bestanden sie, wenn auch nur in ibren Anfängen, schon zur Zeit der Operation, oder sind sie erst nach derselben entstanden und in welchem Umfange? Sicher wird wohl die Annahme berechtigt sein, dass die Degeneration der Pyramidenbahnen schon zur Zeit des operativen Eingriffs sich ausgebildet hatte, spricht doch der Grad der Veränderung hier für ein längeres Bestehen, ebenso dürfte die aufsteigende Degeneration schon zum grösseren Theile vorhanden gewesen sein, dagegen ist die Veränderung in den Hintersträngen unterhalb der Compressionsstelle wohl sicher jüngeren Datums.

Es sind ja in letzter Zeit mehrfach Fälle publicirt worden, wo bei Compression neben den typischen auf- und absteigenden Veränderungen andere constatirt wurden, deren Bedeutung noch keineswegs klar ist. Einmal das von mir gekennzeichnete Gebiet, das an die Pyramidenseitenstrangbahn anschliesst und einen schwächeren Grad der Veränderung zeigt, als letztere selbst, zweitens die zunächst ziemlich ausgedehnte, wenn auch nicht starke Veränderung in den Hintersträngen, die allmälig sich einschränkte, so dass schliesslich nur eine elliptische Figur, die um die hintere Längsspalte gelagert, sich mehr der Peripherie des Markes als der hinteren Commissur aähernd, übrig blieb, beides Degenerationsfelder, die sich unterhalb der Compressionsstelle fanden, würden zu den Abweichungen zu rechnen sein und die Frage gerechtfertigt erscheinen lassen, um welche Fasern handelt es sich hier, kommt ihr Ausfall auch im klinischen Bild zum Ausdruck? 